\title{
Análise comparativa de classificadores digitais em imagens do Landsat-8 aplicados ao mapeamento temático
}

\author{
Danilo Francisco Trovo Garofalo(1), Cassiano Gustavo Messias ${ }^{(1)}$, Veraldo Liesenberg ${ }^{(2)}$, \\ Édson Luis Bolfe ${ }^{(3)}$ e Marcos César Ferreira(1)
}

\begin{abstract}
(1)Universidade Estadual de Campinas, Rua João Pandiá Calógeras, oo 51, CEP 13083-870 Campinas, SP, Brasil. E-mail: danilogarofalo@ige.unicamp.br, cassianomessias@ige.unicamp.br, macferre@ige.unicamp.br (2)Universidade do Estado de Santa Catarina, Avenida Luiz de Camões, № 2.090, CEP 88520-000 Lages, SC, Brasil. E-mail: veraldo.liesenberg@udesc.br (3)Embrapa Monitoramento por Satélite, Avenida Soldado Passarinho, no 303, CEP 13070-115 Campinas, SP, Brasil. E-mail: edson.bolfe@embrapa.br
\end{abstract}

Resumo - O objetivo deste trabalho foi avaliar o desempenho dos classificadores digitais SVM e K-NN para a classificação orientada a objeto em imagens Landsat-8, aplicados ao mapeamento de uso e cobertura do solo da Alta Bacia do Rio Piracicaba-Jaguari, MG. A etapa de pré-processamento contou com a conversão radiométrica e a minimização dos efeitos atmosféricos. Em seguida, foi feita a fusão das bandas multiespectrais (30 m) com a banda pancromática $(15 \mathrm{~m})$. Com base em composições RGB e inspeções de campo, definiramse 15 classes de uso e cobertura do solo. Para a segmentação de bordas, aplicaram-se os limiares 10 e 60 para as configurações de segmentação e união no aplicativo ENVI. A classificação foi feita usando SVM e K-NN. Ambos os classificadores apresentaram elevados valores de índice Kappa (k): 0,92 para SVM e 0,86 para K-NN, significativamente diferentes entre si a $95 \%$ de probabilidade. Uma significativa melhoria foi observada para SVM, na classificação correta de diferentes tipologias florestais. A classificação orientada a objetos é amplamente aplicada em imagens de alta resolução espacial; no entanto, os resultados obtidos no presente trabalho mostram a robustez do método também para imagens de média resolução espacial.

Termos para indexação: classificação orientada a objetos, gestão territorial, sensoriamento remoto, resolução espacial, uso e cobertura do solo.

\section{Comparative analysis of digital classifiers of Landsat-8 images for thematic mapping procedures}

\begin{abstract}
The objective of this work was to evaluate the performance of SVM and K-NN digital classifiers for the object-based classification on Landsat-8 images, applied to mapping of land use and land cover of Alta Bacia do Rio Piracicaba-Jaguari, in the state of Minas Gerais, Brazil. The pre-processing step consisted of using radiometric conversion and atmospheric correction. Then the multispectral bands $(30 \mathrm{~m})$ were merged with the panchromatic band $(15 \mathrm{~m})$. Based on RGP compositions and field inspection, 15 land-use and land-cover classes were defined. For edge segmentation, the bounds were set to 10 and 60 for segmentation configuring and merging in the ENVI software. Classification was done using SVM and K-NN. Both classifiers showed high values for the Kappa index (k): 0.92 for SVM and 0.86 for K-NN, significantly different from each other at $95 \%$ probability. A major improvement was observed for SVM by the correct classification of different forest types. The object-based classification is largely applied on high-resolution spatial images; however, the results of the present work show the robustness of the method also for medium-resolution spatial images.

Index terms: object-based classification, territorial management, remote sensing, spatial resolution, land use and land cover.
\end{abstract}

\section{Introdução}

A gestão territorial demanda uma constante caracterização dos recursos naturais, além de seu monitoramento contínuo, com o objetivo de sua utilização de forma racional. A execução de projetos de levantamento e mapeamento da superfície terrestre têm-se beneficiado do avanço nas áreas de sensoriamento remoto e geoprocessamento. Com o advento dos sensores orbitais, principalmente a partir da série de satélites Landsat, iniciada em 1972, grandes áreas puderam ser caracterizadas e monitoradas, o que possibilita a realização de estudos em extensas regiões a custo relativamente baixo (Jensen, 2005).

Em geral, o processo de classificação pode ser feito por interpretação visual ou por classificação digital. Para 
a interpretação visual, o fotointérprete extrai feições de interesse das imagens, em geral uma composição colorida RGB. Já a classificação digital é um processo de reconhecimento de padrões e de objetos homogêneos, representados em um conjunto de pixels, ao qual se aplica o mapeamento de áreas pertencentes a uma única classe de objetos (Jensen, 2005). Ainda, segundo este autor, os algoritmos responsáveis pela classificação digital são denominados "classificadores", e a classificação pode ser feita pixel a pixel ou por regiões/objetos.

As classificações pixel a pixel baseiam-se principalmente em parâmetros estatísticos (média, variância etc.) dos pixels de treinamento, a exemplo dos perfis espectrais, para o agrupamento dos pixels remanescentes nas classes predeterminadas (Jensen, 2005). As classificações por região ou orientadas a objetos, do inglês "object-based image analysis" (OBIA), utilizam, além de informação espectral de cada pixel, a informação espacial que envolve a relação de pixels circunvizinhos, tais como forma e textura (Ponzoni et al., 2012). Assim, o classificador simula a análise de um fotointérprete, ao delimitar áreas homogêneas nas imagens, a partir das características espectrais e espaciais dos objetos que constituem as classes de interesse (Ponzoni et al., 2012).

Uma gama de classificadores, aplicados à classificação supervisionada pixel a pixel, tem sido amplamente empregada na classificação de imagens remotamente situadas, e os classificadores Mahalanobis, "maximum likelihood", "minimum distance" e "parellelepiped" têm sido os mais utilizados (Sisodia et al., 2014), todos de natureza paramétrica. Neste contexto, muitos trabalhos têm avaliado a acurácia desses classificadores, para a definição de áreas homogêneas a partir de imagens de resolução espacial moderada (Hagner \& Reese, 2007; Prishchepov et al., 2012; Laurin et al., 2013; Jia et al., 2014; Ganasri \& Dwarakish, 2015).

Recentemente, inúmeros trabalhos têm mostrado que técnicas de classificação não paramétricas, com o emprego de classificadores "máquinas de vetores-suporte ("support vector machine", SVM) e "k-vizinhos mais próximos" ("k-nearest neighbor", K-NN), têm-se destacado na classificação orientada a objetos (Mountrakis et al., 2011; Dronova et al., 2012; Liu et al., 2013; Poursanidis et al., 2015; Wahidin et al., 2015), uma vez que atributos como forma, textura e nitidez apresentam natureza não paramétrica.

Em atividade há mais de 40 anos, a série Landsat conta atualmente com o satélite Landsat-8. Contudo, ainda há carência de estudos que visem avaliar comparativamente a acurácia de classificadores digitais, aplicados a classificações orientadas a objetos, a partir de imagens Landsat-8 adquiridas em ambientes tropicais.

O objetivo deste trabalho foi avaliar o desempenho dos classificadores digitais SVM e K-NN para a classificação orientada a objeto em imagens Landsat-8, aplicados ao mapeamento de uso e cobertura do solo da Alta Bacia do Rio Piracicaba-Jaguari, MG.

\section{Material e Métodos}

A área de estudo compreende a Alta Bacia do Rio Piracicaba-Jaguari (PJ), no Estado de Minas Gerais. Com área de drenagem de aproximadamente $1.158 \mathrm{~km}^{2}$, a bacia abrange os municípios de Extrema, Itapeva e Toledo e parte dos municípios de Camanducaia e Sapucaí-Mirim, na região sul de Minas Gerais, junto à divisa com o Estado de São Paulo (Figura 1).

Situada na região da Mantiqueira Meridional e inserida nos planaltos de Campos do Jordão e Lindóia, a bacia PJ apresenta altitudes que variam entre $833 \mathrm{~m}$ e 2.032 m (Apa..., 1998). De acordo com a classificação climática de Köppen, a bacia PJ está sob domínio Cwb - clima temperado úmido, com inverno seco e verão temperado.

As condições topoclimáticas da bacia PJ influenciam a composição florística e a distribuição de suas formações vegetais, compostas por remanescentes florestais de Floresta Estacional Semidecídua, Floresta Ombrófila Densa Atlântica e alguns dos últimos e significativos fragmentos de Floresta Ombrófila Mista do Brasil (Moretti, 2011), além de áreas de regeneração, representadas pela capoeira. Contudo, essas coberturas vegetais têm sido gradativamente substituídas por atividades agropastoris e silvicultura.

As atividades agropastoris são representadas principalmente por cultivos agrícolas temporários, dos quais se destacam batata, feijão, milho e repolho, e por pastagens preparadas para pecuária de forma extensiva. A silvicultura é representada pelo cultivo de eucalipto e pinus. Observamos, ainda, um adensamento urbano no eixo da Rodovia Fernão Dias (Figura 1), além das áreas urbanas do Município de Toledo e do Distrito de Monte Verde.

Para o desenvolvimento da pesquisa, utilizou-se uma imagem do sensor OLI/LDCM (Landsat-8), órbita/ ponto $219 / 76$, com data de passagem do satélite em 
$1^{\circ}$. de setembro de 2013. O sensor OLI, em órbita no LDCM, tem resolução temporal de 16 dias, resolução radiométrica de 12 bits, escalonados posteriormente para 16 bits, e apresenta nove diferentes bandas espectrais que têm diferentes características espectrais e espaciais (Frequently..., 2014). No presente trabalho, foram empregadas oito das nove bandas espectrais (Tabela 1).

Diferentes etapas de pré-processamento foram aplicadas. A correção geométrica não foi necessária, pois as imagens já são fornecidas com georreferenciamento.

As bandas multiespectrais e pancromática foram inicialmente convertidas em radiância $\left(\mathrm{W} \times \mathrm{m}^{-2} \times \mathrm{sr}^{-1} \times \mathrm{nm}^{-1}\right)$, por meio do uso de valores de ganho e off-set fornecidos com os metadados da imagem. A minimização dos efeitos atmosféricos, nas imagens Landsat-8, foi feita com o módulo "fast line-of-sight atmospheric analysis of spectral hypercubes" (Flaash) (Felde et al., 2003). Para o procedimento da correção atmosférica, foram utilizados os filtros de sensitividade espectral das bandas multiespectrais e pancromática, visibilidade de $85 \mathrm{~km}$, coluna d'água de $3,288 \mathrm{~g} \mathrm{~cm}^{-2}$, altitude média da área de $1.530 \mathrm{~m}$. Considerou-se como modelos de atmosfera e de aerossóis o tropical e o rural, respectivamente. Como resultados, os valores de reflectância de superfície (fator de reflectância direcional hemisférico) para as bandas multiespectrais e pancromática foram obtidos. Uma descrição mais detalhada sobre a metodologia, bem como a terminologia empregada estão disponíveis em Oliveira et al. (2009).

Após a correção atmosférica, a fusão das bandas multiespectrais (bandas espectrais de 1 a 7, Tabela 1), com resolução espacial de $30 \mathrm{~m}$, à banda pancromática (banda 8, Tabela 1) com resolução espacial de $15 \mathrm{~m}$ foi realizada. Para isso, usou-se o comando "Gram-Schmidt spectral sharpening" e o método "average of low resolution multiespectral file". Como produto final, bandas multiespectrais com resolução espacial de $15 \mathrm{~m}$ foram obtidas.

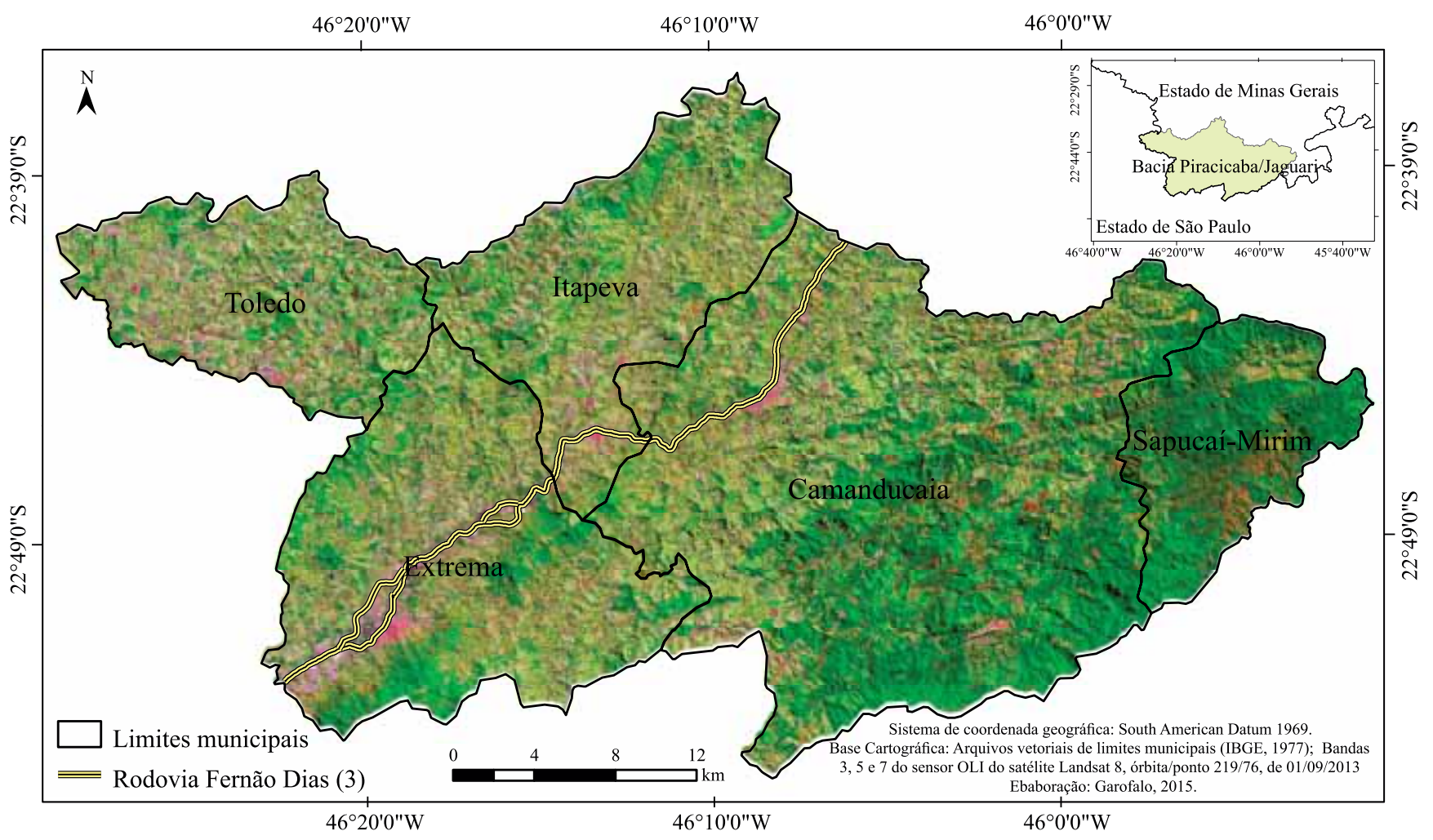

Figura 1. Mapa de localização da Alta Bacia do Rio Piracicaba-Jaguari, MG, e limites territoriais dos municípios nela inseridos, sobre composição colorida falsa-cor R7G5B3 — Landsat-8. 
Com base nos dados supracitados em uma composição falsa-cor (i.e. R5G4B6) e inspeções de campo, foram definidas 15 classes de uso e cobertura do solo. Os remanescentes florestais foram divididos em quatro classes distintas, de acordo com suas características fisiológicas e estruturais: capoeira, Floresta Estacional Semidecídua, Floresta Ombrófila Densa e Floresta Ombrófila Mista. As áreas de uso antrópico foram divididas em oito classes: mancha urbana, cultivos agrícolas temporários, pastagem com baixo teor de fitomassa, pastagem com alto teor de fitomassa, eucalipto, pinus, solo exposto destinado à agricultura e solo exposto destinado à silvicultura. Outras três classes foram definidas: afloramento de rocha, corpos d'água e sombra.

Para a geração dos produtos finais da pesquisa, usou-se o módulo "Envi feature extraction" (Envi FX), que possibilita a extração de informações e classificação de imagens com base em características espectrais, espaciais e de textura (Classificação..., 2014). Inicialmente, aplicou-se a segmentação da imagem em regiões homogêneas. Nesse processo, as bandas multiespectrais do Landsat- 8 fusionadas à banda pancromática foi utilizada.

O processo de segmentação apresenta dois processos principais, o de segmentação propriamente dita denominado "segment setting" -, e o "merge setting". A segmentação é um método que reparte uma imagem em inúmeros segmentos. Por meio de parâmetros de entrada predefinidos, é feito o agrupamento de pixels circunvizinhos que apresentam semelhança em suas feições como textura, forma, cor etc. (Classificação..., 2014).

O Envi FX apresenta dois algoritmos para fazer a segmentação: "edge" e "intensity". Com base no

Tabela 1. Características das imagens obtidas pelas diferentes bandas do sensor OLI-LDCM.

\begin{tabular}{lcc}
\hline Banda & $\begin{array}{c}\text { Resolução } \\
\text { espacial }(\mathrm{m})\end{array}$ & $\begin{array}{c}\text { Resolução } \\
\text { espectral }(\mu \mathrm{m})\end{array}$ \\
\hline Banda 1, costeira & 30 & $0,433-0,453$ \\
Banda 2, azul & 30 & $0,450-0,515$ \\
Banda 3, verde & 30 & $0,525-0,600$ \\
Banda 4, vermelho & 30 & $0,630-0,680$ \\
Banda 5, infravermelho próximo & 30 & $0,845-0,885$ \\
Banda 6, infravermelho médio & 30 & $1,560-1,660$ \\
Banda 7, infravermelho médio & 30 & $2,100-2,300$ \\
Banda 8, pancromático & 15 & $0,500-0,680$ \\
\hline
\end{tabular}

Fonte: adaptado de Frequently... (2014). valor estipulado pelo usuário por meio da escala de segmentação, o algoritmo elimina as bordas dos segmentos mais "fracos" e obtém um resultado que pode variar entre segmentos mais suaves ou mais robustos (Classificação..., 2014). Ainda, a etapa "merge" dos segmentos é empregada para agregar segmentos pequenos em regiões maiores (Classificação..., 2014). Aplicou-se os limiares 10 para o item "segment setting", com aplicação do algoritmo "edge", e 60 para "merge setting", ambos escolhidos por tentativa e erro, até a obtenção de um resultado satisfatório verificado de forma visual sobre composição falsa-cor R5G4B6. As feições delineadas no processo de segmentação foram exportadas para um arquivo vetorial.

A partir do arquivo segmentado supracitado, sobreposto à composição R5G4B6, 100 polígonos - ou seja, regiões de interesse (ROI) - foram extraídos para cada uma das classes de forma visual e usados como dados de treinamento.

Foram feitas então as classificações, com aplicação dos algoritmos SVM e K-NN, que estão disponíveis no Envi FX. Nesta etapa, cada feição do arquivo gerado na segmentação é classificada com base nas características espectrais, de forma e de textura de cada classe, de acordo com os dados de treinamento.

O SVM é um método computacional de aprendizado para problemas de reconhecimento de padrão, com base no princípio de separação ótima entre classes. A partir das amostras de treinamento, há uma distribuição de probabilidade. Após a remoção de algumas amostras, são verificadas as mesmas distribuições de probabilidade repetidamente, para que sejam estabelecidos, a posteriori, vetores de suporte em um hiperplano n-dimensional (Huang et al., 2002). A separação ótima se dá pela geração de um hiperplano condicional, baseado nos vetores de suporte supracitados, orientado de forma a englobar o conjunto de dados de cada classe, para maximizar a margem e o ponto mais próximo de cada uma das classes predefinidas (Huang et al., 2002). Um maior detalhamento do método SVM, bem como aplicações na área do sensoriamento remoto podem ser encontradas em Melgani \& Bruzzone (2004), Waske et al. (2010) e Liesenberg \& Gloaguen (2013).

O algoritmo K-NN é uma classificação feita a partir das amostras de treinamento, com base nos vizinhos mais próximos (Meng et al., 2007). Devese atribuir um valor $\mathrm{k}$, que é o número de vizinhos a serem utilizados na determinação da classe que será 
atribuída pelos valores de reflectância de superfície da maioria dos pixels circunvizinhos (Meng et al., 2007). Um maior detalhamento do método K-NN, bem como aplicações na área do sensoriamento remoto podem ser encontrados em Meng et al. (2007), Samaniego \& Schulz (2009) e Vibrans et al. (2013).

A acurácia dos mapas temáticos gerados foi calculada, a fim de constatar qual classificador gerou os melhores resultados. Os dois métodos utilizados tratam da exatidão global (EG) (Congalton \& Green, 1999) e do índice Kappa (k) (Congalton \& Green, 1999). Foram delineadas 20 amostras de referência para cada uso, compostas por 50 pixels cada, por inspeção e interpretação visual por meio do Google Earth (defasagem temporal inferior a um ano) e por meio de imagens da constelação RapidEye do ano de 2013. Dados de inspeções de campo, realizado no ano de 2013, auxiliaram na escolha das amostras de referência.

Assim, na primeira etapa, avaliou-se o desempenho das classificações, a partir dos valores Kappa obtidos e do conceito de desempenho listados a seguir, de acordo com o estabelecido por Congalton \& Green (1999):
$\mathrm{K} \leq 0$, péssimo; $0<\mathrm{K} \leq 0,2$, ruim; $0,2<\mathrm{K} \leq 0,4$, razoável; $0,4<\mathrm{K} \leq 0,6$, bom; $0,6<\mathrm{K} \leq 0,8$, muito bom; $0,8<\mathrm{k}<1$, excelente.

Em segunda etapa, avaliou-se o desempenho entre classificadores ao nível de significância de 95\%, com a aplicação do teste Z (Congalton \& Green, 1999): $\mathrm{z}=\mathrm{K}_{2}-\mathrm{K}_{1} /\left(\sigma_{\mathrm{k}_{2}}^{2}+\sigma_{\mathrm{k}_{1}}^{2}\right)^{0,5}$, em que $\mathrm{K}_{1}$ é o índice Kappa da imagem 1; $\mathrm{K}_{2}$ é o índice Kappa da imagem 2; e $\sigma^{2}$ é a variância do índice Kappa. Quando z >1,96 (95\%), o teste é significativo, e a hipótese de nulidade é rejeitada, portanto, podemos concluir que há diferença estatística entre as classificações.

Por fim, analisou-se as áreas em discordância entre os resultados gerados, com o propósito de avaliar se tais diferenças entre classificadores derivam não somente da confusão espectral, mas também de influências das características fisiográficas locais como a densidade da drenagem (Figura 2). Para tanto, subtraímos a classe A, classificada pelo SVM, da classe A classificada pelo K-NN e, posteriormente, subtraímos a classe A, classificada pelo K-NN, da classe A, classificada pelo SVM. Os produtos das subtrações foram somados e, como resultado, as áreas em discordância para a classe

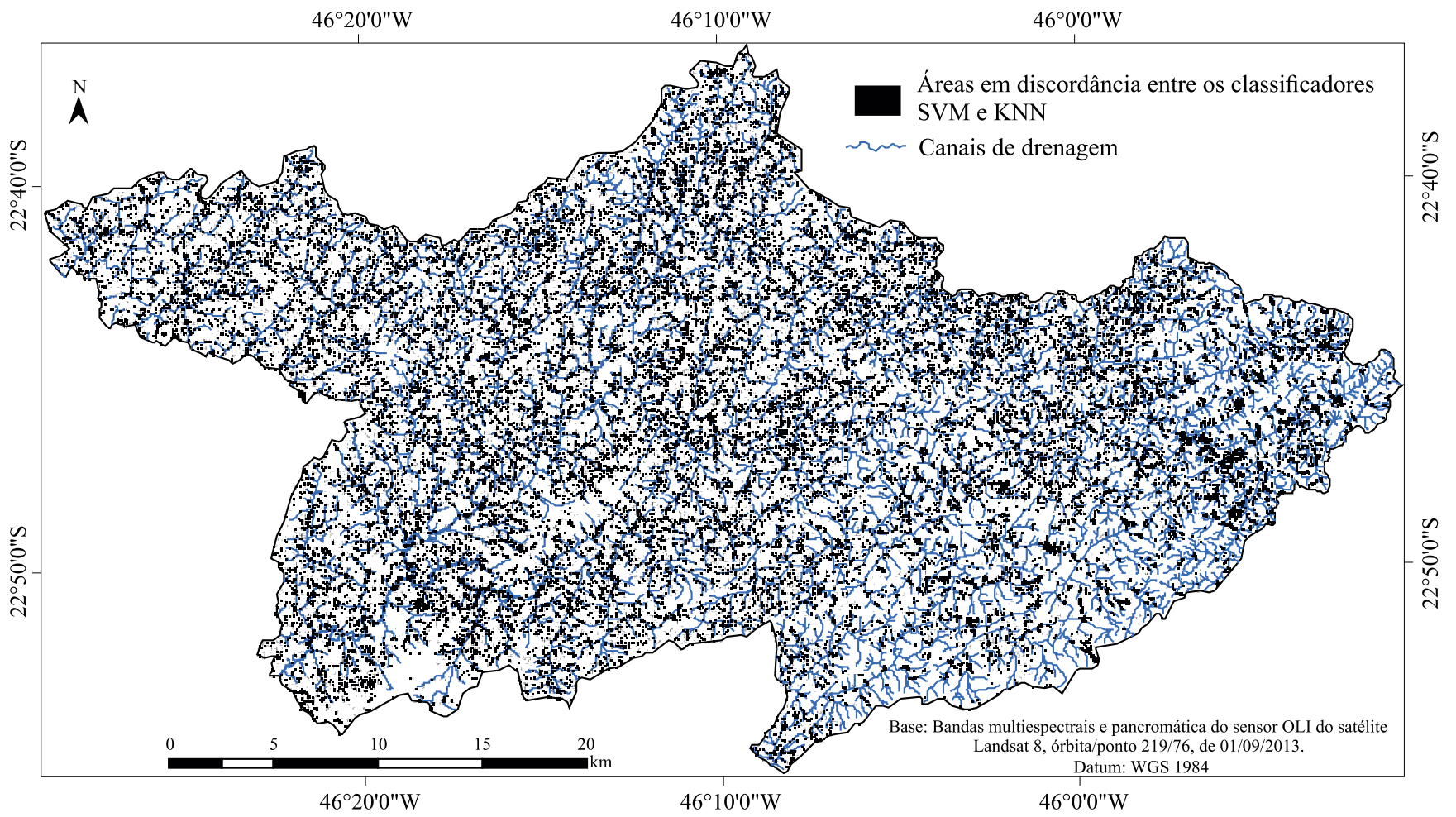

Figura 2. Mapa de áreas em discordância entre as classificações digitais SVM e K-NN na Alta Bacia do Rio PiracicabaJaguari, MG. 
A foram obtidas. $\mathrm{O}$ mesmo procedimento foi realizado para as demais classes de uso e cobertura do solo. Por fim, as áreas em discordância de todas as classes foram somados, e o produto desta soma representa as áreas em discordância entre os resultados dos dois classificadores.

\section{Resultados e Discussão}

Em geral, os perfis espectrais das 15 classes de uso e cobertura do solo apresentados na Figura 3 corroboram aqueles reportados em Galvão et al. (2009), Liesenberg et al. (2010) e Ponzoni et al. (2012). Uma análise desses perfis espectrais, mostra que uma classificação pautada apenas nas características espectrais dos objetos tenderia a apresentar inconsistências entre classes com perfis espectrais semelhantes. Evidencia-se, portanto, a importância de também serem considerados os atributos referentes à forma e à textura dos objetos analisados. A partir da segmentação realizada, foram obtidos diferentes resultados nos mapas temáticos gerados, de acordo a aplicação de cada algoritmo utilizado na classificação (Figura 4).

As classificações pelos métodos SVM e K-NN apresentaram percentagens semelhantes das áreas ocupadas pelas classes de uso e cobertura selecionadas (Tabela 2). Segundo os classificadores, a bacia PJ tem aproximadamente $50 \%$ de sua área coberta por remanescentes florestais, $25 \%$, por pastagens, e $25 \%$ pelas demais classes.

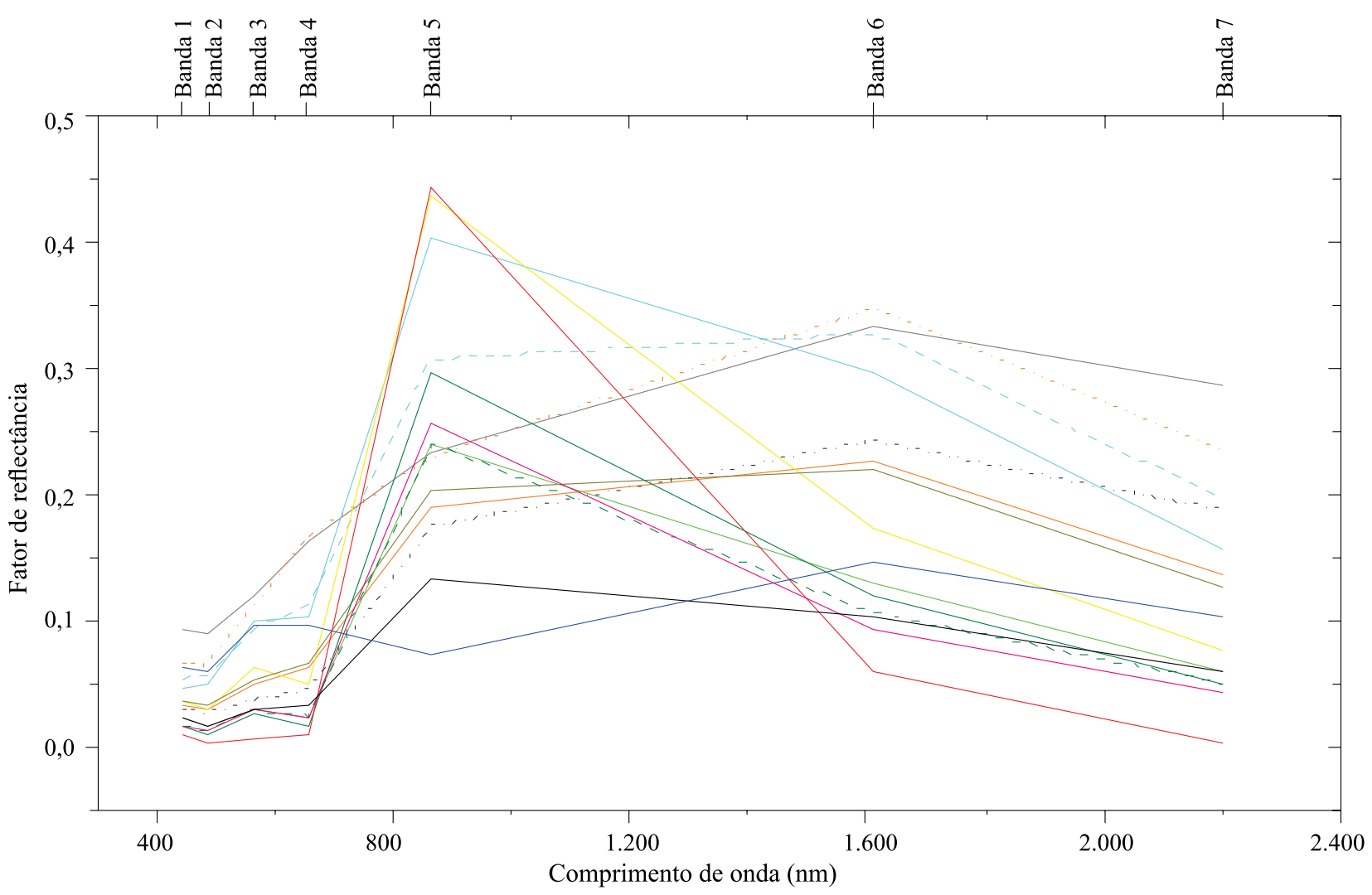

\begin{tabular}{|c|c|c|}
\hline Mancha urbana & Cultivos agrícolas & Eucalipto \\
\hline Solo silvicultura & Capoeira & Pinus \\
\hline Solo agricultura & Floresta Estacional Decídua & Sombra - vegetação \\
\hline Pastagem com baixa fitomassa & Floresta Ombrófila Mista & Afloramento de rocha \\
\hline Pastagem com alta fitomassa & Floresta Ombrófila Densa & Corpos d'água \\
\hline
\end{tabular}

Figura 3. Perfil espectral das classes de uso do solo identificadas na Alta Bacia Piracicaba-Jaguari, MG. 
Os classificadores SVN e K-NN obtiveram (Congalton \& Green, 1999). Contudo, os valores desempenho excelente $(Z=5,68)$ segundo o teste $Z$ obtidos pelo teste $Z$ (Tabela 3) evidenciam uma
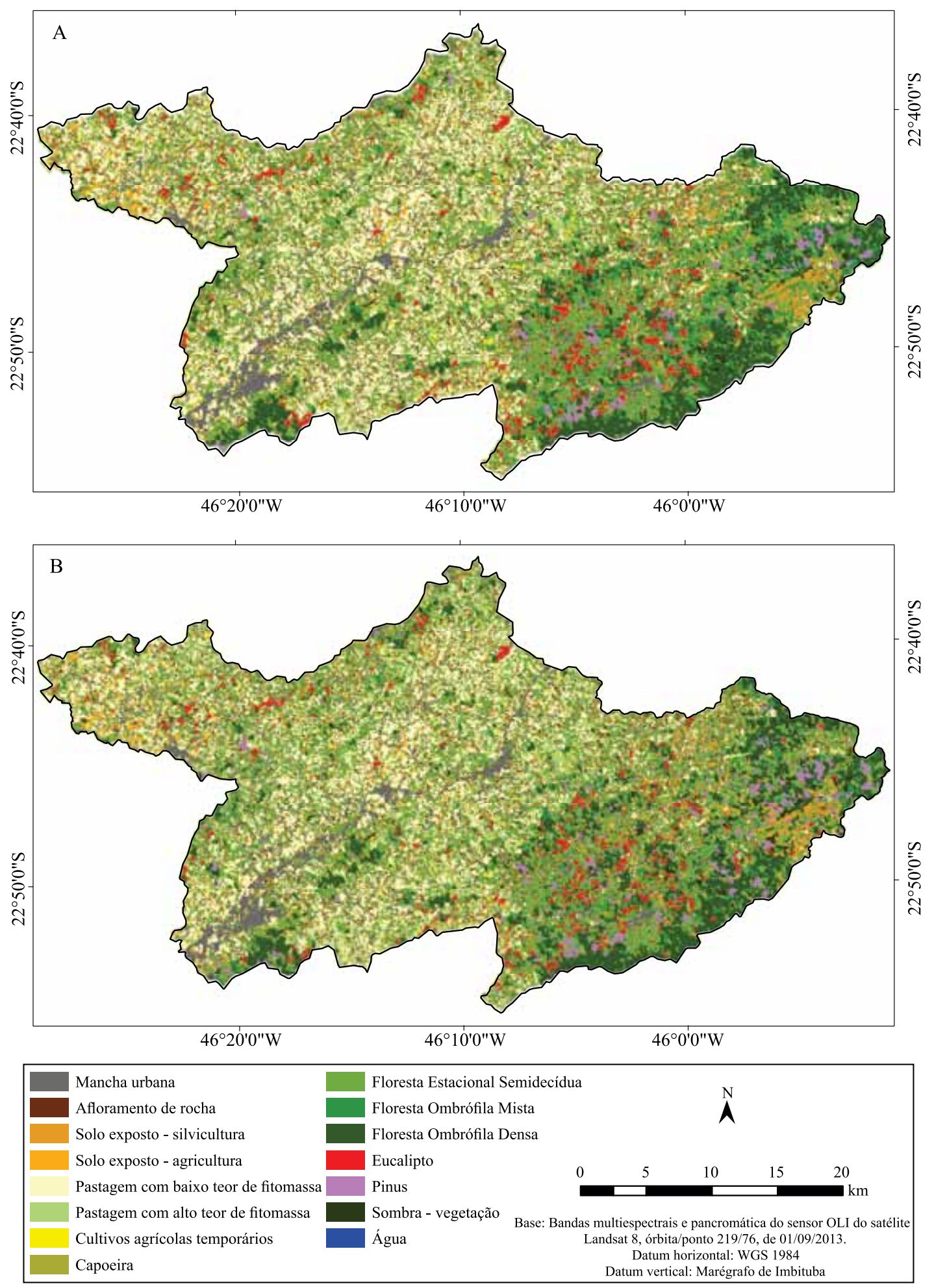

Figura 4. Mapas de uso do solo e cobertura vegetal da Alta Bacia do Rio Piracicaba-Jaguari, MG, segundo os classificadores digitais SVM (A) e K-NN (B) para classificação orientada a objeto. 
diferença sutil entre as classificações SVM/K-NN a $95 \%$ de probabilidade $(Z=1,96)$.

A validação dos resultados, pelo método de exatidão global, mostra que o classificador que apresentou melhor acurácia foi o SVM, com valor 0,93 , enquanto o K-NN obteve o valor 0,88 . Esses valores corroboram aqueles obtidos pelo índice Kappa, de 0,92, e 0,86, respectivamente.

O classificador SVM foi o que apresentou melhor desempenho, com acerto superior a $90 \%$ para 12 das 15 classes (Tabela 3 A), e obteve os melhores resultados para as classes pinus, sombra, cultivo agrícola, pastagem com alto teor de fitomassa e área urbana. As maiores inconsistências foram observadas para as classes de solo destinado à agricultura, eucalipto e solo destinado à silvicultura.

Assim como nas inconsistências de classificação observadas para o método K-NN, as inconsistências do classificador SVM também estão relacionadas à similaridade espectral e textural dos objetos, de modo que solos destinados à silvicultura foram classificados como capoeira $(25,7 \%)$. A capoeira é uma vegetação secundária, composta predominantemente por gramíneas e arbustos esparsos com exposição de folhas secas e solo. $\mathrm{O}$ solo destinado à silvicultura tende a apresentar resíduos e serapilheira (restos da colheita) sobre o solo, o que lhe confere um comportamento espectral semelhante ao da capoeira. Eucalipto foi

Tabela 2. Área das classes identificadas e suas proporções em percentagem, de acordo com os classificadores SVM e $\mathrm{K}-\mathrm{NN}$.

\begin{tabular}{lccccc}
\hline Classe & \multicolumn{2}{c}{ Área $\left(\mathrm{km}^{2}\right)$} & & Percentagem $(\%)$ \\
\cline { 2 - 3 } \cline { 6 - 6 } & SVM & $\mathrm{KNN}$ & & SVM & $\mathrm{KNN}$ \\
\hline Área urbana & 65,94 & 71,65 & & 5,7 & 6,19 \\
Solo destinado à silvicultura & 21,95 & 40,2 & 1,9 & 3,47 \\
Solo destinado à agricultura & 27,35 & 30,45 & 2,36 & 2,63 \\
Pastagem com baixo teor de fitomassa & 244,7 & 204,48 & 21,14 & 17,66 \\
Pastagem com alto teor de fitomassa & 59,51 & 80,24 & 5,14 & 6,93 \\
Cultivos agrícolas & 41,46 & 34,32 & 3,58 & 2,97 \\
Capoeira & 43,78 & 46,71 & 3,78 & 4,04 \\
Floresta Ombrófila Mista & 55,17 & 44,19 & 4,77 & 3,82 \\
Floresta Estacional Semidecídua & 379,12 & 367,1 & 32,75 & 31,71 \\
Floresta Ombrófila Densa & 97,6 & 110,54 & 8,43 & 9,55 \\
Eucalipto & 41,92 & 35,57 & 3,62 & 3,07 \\
Pinus & 13,64 & 24,52 & 1,18 & 2,12 \\
Afloramento de rocha & 52,16 & 49,17 & 4,51 & 4,25 \\
Sombra & 12,04 & 13,82 & 1,04 & 1,19 \\
Corpos d'água & 1,2 & 4,59 & 0,1 & 0,4 \\
\hline Total & $1.157,55$ & \multicolumn{3}{c}{100} \\
\hline
\end{tabular}

classificado como Floresta Ombrófila Mista (20,4\%) e solo destinado à agricultura foi classificado como Floresta Estacional Semidecídua (23\%).

O alto desempenho do SVM, com baixo erro de classificação na separabilidade de coberturas florestais distintas, se deve à robustez e capacidade de formar planos n-dimensionais deste método.

As melhores qualidades em classificação, obtidas pelo método K-NN, foram encontradas nas seguintes classes: área urbana, pastagens, cultivos agrícolas, capoeira, eucalipto, água, Floresta Ombrófila Mista, sombra, pinus e solo destinado à agricultura, com acertos próximos ou superiores a $80 \%$ (Tabela 3 B). Entre estas classes, observou-se maior erro de classificação entre áreas de pastagem com alto teor de fitomassa classificadas como aquelas de baixo teor de fitomassa $(11,3 \%)$ e cultivos agrícolas $(8,1 \%)$, assim como áreas de eucalipto classificadas como pinus $(9,8 \%)$. Tais inconsistências podem estar associadas ao comportamento espectral semelhante dessas classes, que tendem a influenciar até mesmo uma classificação orientada a objetos.

Classes como solo destinado à silvicultura e Floresta Estacional Semidecídua apresentaram os menores acertos pelo classificador K-NN, com valores de 60,6 e $54,9 \%$, respectivamente (Tabela $3 \mathrm{~B}$ ). Na região do visível $(0,433-0,68 \mu \mathrm{m})$, a classe Floresta Estacional Semidecídua apresentou comportamento espectral muito semelhante ao das classes pinus, sombra, Floresta Ombrófila Densa e capoeira, porém distinto na região do infravermelho $(0,845-2,3 \mu \mathrm{m})$. Assim, o maior número de bandas na região do visível pode ter influenciado as inconsistências de classificação da classe Floresta Estacional Semidecídua com as classes supracitadas. Vale ressaltar também a condição sazonal dessa cobertura vegetal quanto ao período do imageamento, no mês de setembro, quando há perda de grande parte das folhas e consequente alteração do comportamento espectral do dossel, o que torna a sua textura mais rugosa, similar à dos dosséis das classes que foram confundidas com Floresta Estacional Semidecídua. Neste caso, o uso de uma imagem em outra data poderia melhorar, de forma combinada ou isolada, eventualmente, a acurácia desta classe.

Uma análise mais qualitativa entre as diferenças apresentadas entre as classificações obtidas pelos métodos SVM e K-NN foi possibilitada pela espacialização das áreas em discordância entre os resultados gerados. Os pixels corretos (sejam no SVM e K-NN) são ilustrados na cor branca. Os pixels que 
apresentaram discordância estão representados na cor preta (Figura 2).

Grandes manchas de discordância na porção sudeste da bacia, relacionadas a áreas de remanescentes florestais classificados diferentemente entre os classificadores SVM e K-NN foram observados (Figura 4). Uma análise desse mapa, em conjunto com as matrizes de erro desses classificadores, evidencia a superioridade do classificador SVM para separar diferentes coberturas florestais, principalmente entre áreas classificadas como pinus, pelo classificador SVM, e Floresta Ombrófila Mista, pelo classificador K-NN, uma vez que o SVM obteve $100 \%$ de acerto na classificação de pinus, e o K-NN obteve acerto de $82,2 \%$ na classificação da classe Floresta Ombrófila Mista quando, de acordo com as amostras de referência, $11,1 \%$ das áreas classificadas erroneamente nessa classe correspondem a pinus.

A maior concentração de áreas em discordância ocorreu na porção centro-oeste da bacia, no corredor de

Tabela 3. Matrizes de erro dos classificadores SVM (parte superior) e K-NN (parte inferior), para as diferentes classes de uso e cobertura do solo na Bacia Piracicaba-Jaguari, $\mathrm{MG}^{(1)}$.

\begin{tabular}{|c|c|c|c|c|c|c|c|c|c|c|c|c|c|c|c|}
\hline Classe & $\mathrm{A}$ & $\mathrm{B}$ & $\mathrm{C}$ & $\mathrm{D}$ & $E$ & $\mathrm{~F}$ & $\mathrm{G}$ & $\mathrm{H}$ & $\mathrm{I}$ & $\mathrm{J}$ & $\mathrm{K}$ & $\mathrm{L}$ & $\mathrm{M}$ & $\mathrm{N}$ & $\mathrm{O}$ \\
\hline $\mathrm{A}$ & 99,8 & - & - & 0,2 & - & - & - & - & - & - & - & - & - & - & - \\
\hline B & - & 72,3 & - & - & - & 25,7 & - & - & - & - & 2,0 & - & - & - & - \\
\hline $\mathrm{C}$ & - & - & 93,9 & - & - & 6,1 & - & - & - & - & - & - & - & - & - \\
\hline D & - & - & - & 98,2 & 1,8 & - & - & - & - & - & - & - & - & - & - \\
\hline $\mathrm{E}$ & - & - & - & 3,9 & 96,1 & - & - & - & - & - & - & - & - & - & - \\
\hline $\mathrm{F}$ & - & 5,8 & - & - & - & 94,2 & - & - & - & - & - & - & - & - & - \\
\hline G & - & - & - & - & - & - & 71,0 & 8,6 & - & 20,4 & - & - & - & - & - \\
\hline $\mathrm{H}$ & - & - & - & - & - & - & - & - & - & 0,6 & - & - & - & - & 2,5 \\
\hline I & - & - & - & - & - & - & - & - & 91,2 & 8,8 & - & - & - & - & - \\
\hline $\mathrm{J}$ & - & - & - & - & - & - & - & - & - & 94,5 & - & - & - & - & - \\
\hline K & - & - & - & - & - & - & - & - & - & - & 93,6 & - & 6,4 & - & - \\
\hline $\mathrm{L}$ & - & 4,3 & - & - & - & - & - & - & - & - & - & 92,5 & 3,2 & - & - \\
\hline M & - & - & - & - & - & - & - & - & - & - & - & - & 100 & - & - \\
\hline $\mathrm{N}$ & - & - & - & - & - & - & - & - & - & - & - & - & - & 100 & - \\
\hline $\mathrm{O}$ & - & - & - & - & - & - & 5,4 & - & - & 10,8 & 23,0 & - & - & - & 60,8 \\
\hline Acurácia do produtor (\%) & 100 & 91,3 & 100 & 91,5 & 98,7 & 61,3 & 83,5 & 94,9 & 100 & 66,2 & 87,4 & 100 & 82,9 & 100 & 91,8 \\
\hline \multirow[t]{2}{*}{ Acurácia do usuário (\%) } & 99,8 & 72,3 & 93,9 & 98,2 & 96,1 & 94,2 & 71,0 & 94,3 & 91,2 & 94,5 & 93,6 & 92,5 & 100 & 100 & 60,8 \\
\hline & \multicolumn{8}{|c|}{ Exatidão global de 0,933} & \multicolumn{7}{|c|}{ Índice kappa de 0,919} \\
\hline A & 99,8 & - & - & 0,2 & - & - & - & - & - & - & - & - & - & - & - \\
\hline B & - & 60,6 & - & - & - & 20,2 & - & - & - & - & - & - & - & - & - \\
\hline $\mathrm{C}$ & - & 1,2 & 85,4 & 6,1 & - & 7,3 & - & - & - & - & - & - & - & - & - \\
\hline D & - & - & 11,3 & 79,0 & 8,1 & - & 1,6 & - & - & - & - & - & - & - & - \\
\hline E & - & - & - & 4,1 & 95,9 & - & - & - & - & - & - & - & - & - & - \\
\hline $\mathrm{F}$ & - & 22,0 & - & - & - & 78,0 & - & - & - & - & - & - & - & - & - \\
\hline G & - & - & - & - & - & - & 89,6 & - & - & - & - & 0,6 & - & 9,8 & - \\
\hline $\mathrm{H}$ & - & 5,3 & - & - & - & 2,6 & - & 75,4 & 12,3 & - & 2,6 & - & 1,8 & - & - \\
\hline I & - & - & - & - & - & - & - & - & 100 & - & - & - & - & - & - \\
\hline $\mathrm{J}$ & - & - & - & - & - & - & - & - & - & 82,2 & 6,7 & - & - & 11,1 & - \\
\hline K & - & - & - & - & - & 4,9 & - & - & - & - & 54,9 & 7,8 & 17,6 & 14,7 & - \\
\hline $\mathrm{L}$ & - & - & - & - & - & & 9,4 & - & - & 5,9 & 5,9 & 71,3 & 3,0 & 4,5 & - \\
\hline $\mathrm{M}$ & - & - & - & - & - & - & - & - & - & - & - & - & 100 & - & - \\
\hline $\mathrm{N}$ & - & - & - & - & - & - & - & - & - & - & 5,3 & 4,3 & - & 90 & - \\
\hline $\mathrm{O}$ & - & - & - & - & - & - & - & - & - & - & - & - & - & - & 100 \\
\hline Acurácia do produtor (\%) & 100 & 75,0 & 90,9 & 83,1 & 93,3 & 57,5 & 88,0 & 100 & 80,0 & 75,5 & 70,9 & 91,7 & 70,2 & 65,4 & 100 \\
\hline Acurácia do usuário (\%) & 99,8 & 60,6 & 85,4 & 79,0 & 95,9 & 78,0 & 89,6 & 75,4 & 100 & 82,2 & 54,9 & 71,3 & 100 & 90,4 & 100 \\
\hline
\end{tabular}

Exatidão global de 0,885

Índice kappa de 0,860

(1) Os valores em linha representam o desempenho do classificador, e as colunas, a referência. A, área urbana; B, solo de silvicultura; C, pastagem com baixo teor de fitomassa; D, pastagem com alto teor de fitomassa; E, cultivos agrícolas; F, capoeira; G, eucalipto; H, afloramento de rocha; I, água; J, Floresta Ombrófila Mista; K, Floresta Estacional Semidecídua; L, Floresta Ombrófila Densa; M, sombra em vegetação; N, pinus; e O, solo de agricultura. 
influência da Rodovia Fernão Dias. Nessa área, o uso do solo é mais fragmentado, em comparação à porção sudeste da bacia. Tal fato, associado à alta densidade de drenagem decorrente da rugosidade do relevo local (Figura 4), acaba por criar muitas faixas de transição entre classes limítrofes à rede de drenagem. Talvez, uma correção da topografia possa diminuir as diferenças observadas entre esses classificadores. Todavia, o trabalho carece de mais investigação a respeito das possíveis influências para tais discordâncias.

Embora a classificação orientada a objetos seja mais amplamente aplicada em imagens de alta resolução espacial (Mallinis et al., 2008; Tansey et al., 2009; Zhou et al., 2009; Machado et al., 2014), os resultados obtidos no presente trabalho mostram a robustez do método também para imagens de média resolução espacial. Essa adequação também decorre da resolução radiométrica de 16 bits das imagens, da correção atmosférica realizada e da fusão entre bandas multiespectrais, de $30 \mathrm{~m}$ de resolução espacial, e a banda pancromática, de $15 \mathrm{~m}$. Assim, além da informação de reflectância, foram obtidos detalhes de cor, forma, textura e nitidez.

Por fim, os resultados evidenciam a potencialidade da classificação orientada a objetos, pelos métodos SVM e K-NN, como subsídio ao planejamento socioambiental de bacias hidrográficas, bem como do setor agropecuário, e ampara decisões de gestores públicos e privados. Mapas acurados de uso e cobertura do solo são importantes para uma correta gestão territorial, por representar resultados fidedignos à realidade da área em estudo. Diminuem, assim, as inconsistências relacionadas à estimativa de áreas das classes mapeadas, tais como remanescentes florestais, tornando mais confiável a análise de Áreas de Preservação Permanentes (APP), por exemplo (Valle Júnior et al., 2010).

\section{Conclusões}

1. A segmentação das imagens Landsat- 8 e as classificações pelos métodos SVM e K-NN são plenamente satisfatórias na diferenciação de 15 regiões homogêneas, previamente definidas, e viabilizam a obtenção de resultados precisos para a caracterização de uso e cobertura do solo, na Alta Bacia do Rio Piracicaba-Jaguari, MG.
2. O classificador máquina de vetores suporte (SVM) apresenta desempenho superior ao $\mathrm{k}$ vizinhos mais próximos $(\mathrm{K}-\mathrm{NN})$;

3. A região de estudo apresenta aproximadamente $50 \%$ de sua área coberta por remanescentes florestais, que abrangem principalmente áreas de mananciais.

4. Embora a classificação orientada a objetos seja mais amplamente aplicada em imagens de alta resolução espacial, os resultados obtidos neste trabalho mostram a robustez do método também para imagens de média resolução espacial

\section{Agradecimentos}

À Fundação de Amparo à Pesquisa do Estado de São Paulo (Fapesp, processos 13/22185-2 e 13/05081-9), pelo apoio financeiro.

\section{Referências}

APA Fernão Dias: plano de gestão ambiental. Belo Horizonte: DER-MG: Ibitu, 1998.

CLASSIFICAÇÃO de imagens orientada a objetos com ENVI FX. Disponível em: <http://www.envi.com.br/index.php/modulos/fx $>$. Acesso em: 2 set. 2014.

CONGALTON, R.G.; GREEN, K. Assessing the accuracy of remotely sensed data: principles and practices. Boca Raton: CRC Press, 1999. 137p.

DRONOVA, I.; GONG, P.; CLINTON, N.E.; WANG, L.; FU, W.; QI, S.; LIU, Y. Landscape analysis of wetland plant functional types: the effects of image segmentation scale, vegetation classes and classification methods. Remote Sensing of Environment, v.127, p.357-369, 2012. DOI: 10.1016/j.rse.2012.09.018.

FELDE, G.W.; ANDERSON, G.P.; COOLEY, T.W.; MATTHEW, M.W.; ADLER-GOLDEN, S.M.; BERK, A.; LEE, J. Analysis of hyperion data with the FLAASH atmospheric correction algorithm. In: INTERNATIONAL GEOSCIENCE AND REMOTE SENSING SYMPOSIUM, 2003, Toulouse. Proceedings. New York: IEEE, 2003. p.90-92. DOI: 10.1109/IGARSS.2003.1293688.

FREQUENTLY asked questions about the Landsat missions. Available at: $<$ http://landsat.usgs.gov/band_designations_landsat_ satellites.php>. Accessed on: 23 May 2014.

GALVÃO, L.S.; PONZONI, F.J.; LIESENBERG, V.; SANTOS, J.R. dos. Possibilities of discriminating tropical secondary succession in Amazônia using hyperspectral and multiangular CHRIS/PROBA data. International Journal of Applied Earth Observation and Geoinformation, v.11, p.8-14, 2009. DOI: 10.1016/j.jag.2008.04.001.

GANASRI, B.P.; DWARAKISH, G.S. Study of land use/land cover dynamics through classification algorithms for Harangi catchment area, Karnataka State, India. Aquatic Procedia, v.4, p.1413-1420, 2015. DOI: 10.1016/j.aqpro.2015.02.183. 
HAGNER, O.; REESE, H. A method for calibrated maximum likelihood classification of forest types. Remote Sensing of Environment, v.110, p.438-444, 2007. DOI: 10.1016/j. rse.2006.08.017.

HUANG, C.; DAVIS, L.S.; TOWNSHEND, J.R.G. An assessment of support vector machines for land cover classification. International Journal of Remote Sensing, v.23, p.725-749, 2002. DOI: 10.1080/01431160110040323.

JENSEN, J.R. Introductory digital image processing: a remote sensing perspective. $3^{\text {rd }}$ ed. Upper Saddle River: Prentice-Hall, 2005. 526p.

JIA, K.; WEI, X.; GU, X.; YAO, Y.; XIE, X.; LI, B. Land cover classification using Landsat 8 operational land imager data in Beijing, China. Geocarto International, v.29, p.941-951, 2014. DOI: $10.1080 / 10106049.2014 .894586$.

LAURIN, G.V.; LIESENBERG, V.; CHEN, Q.; GUERRIERO, L.; DEL FRATE, F.; BARTOLINI, A.; COOMES, D.; WILEBORE, B.; LINDSELL, J.; VALENTINI, R. Optical and SAR sensor synergies for forest and land cover mapping in a tropical site in West Africa. International Journal of Applied Earth Observation and Geoinformation, v.21, p.7-16, 2013. DOI: 10.1016/j.jag.2012.08.002.

LIESENBERG, V.; BOEHM, H.-D.V.; GLOAGUEN, R. Spectral variability and discrimination assessment in a tropical peat swamp landscape using CHRIS/PROBA data. GIScience and Remote Sensing, v.47,p.541-564, 2010. DOI: 10.2747/1548-1603.47.4.541.

LIESENBERG, V.; GLOAGUEN, R. Evaluating SAR polarization modes at L-band for forest classification purposes in eastern Amazon, Brazil. International Journal of Applied Earth Observation and Geoinformation, v.21, p.122-135, 2013. DOI: 10.1016/j.jag.2012.08.016.

LIU, Z.-G.; PAN, Q.; DEZERT, J. A new belief-based K-nearest neighbor classification method. Pattern Recognition, v.46, p.834-844, 2013. DOI: 10.1016/j.patcog.2012.10.001.

MACHADO, C.A.S.; BELTRAME, A.M.K.; SHINOHARA, E.J.; GIANNOTTI, M.A.; DURIEUX, L.; NÓBREGA, T.M.Q.; QUINTANILHA, J.A. Identifying concentrated areas of trip generators from high spatial resolution satellite images using object-based classification techniques. Applied Geography, v.53, p.271-283, 2014. DOI: 10.1016/j.apgeog.2014.06.022.

MALLINIS, G.; KOUTSIAS, N.; TSAKIRI-STRATI, M.; KARTERIS, M. Object-based classification using Quickbird imagery for delineating forest vegetation polygons in a Mediterranean test site. ISPRS Journal of Photogrammetry and Remote Sensing, v.63, p.237-250, 2008. DOI: 10.1016/j. isprsjprs.2007.08.007.

MELGANI, F.; BRUZZONE, L. Classification of hyperspectral remote sensing images with support vector machines. IEEE Transactions on Geoscience and Remote Sensing, v.42, 2004. DOI: $10.1109 /$ TGRS.2004.831865.

MENG, Q.; CIESZEWSKI, C.J.; MADDEN, M.; BORDERS, B.E. K nearest neighbor method for forest inventory using remote sensing data. GIScience and Remote Sensing, v.44, p.149-165, 2007. DOI: $10.2747 / 1548-1603.44 .2 .149$.
MORETTI, A.I.P. Mapeamento de corredores ecológicos na APA Fernão Dias - MG a partir de técnicas de geoprocessamento e análise espacial. 2011. 126p. Dissertação (Mestrado) - Instituto de Geociências, Universidade Estadual de Campinas, Campinas.

MOUNTRAKIS, G.; IM, J.; OGOLE, C. Support vector machines in remote sensing: a review. ISPRS Journal of Photogrammetry and Remote Sensing, v.66, p.247-259, 2011. DOI: 10.1016/j. isprsjprs.2010.11.001.

OLIVEIRA, L.G.L. de; PONZONI, F.J.; MORAES, E.C. Conversão de dados radiométricos orbitais por diferentes metodologias de caracterização atmosférica. Revista Brasileira de Geofísica, v.27, p.121-133, 2009. DOI: 10.1590/S0102-261X2009000100010.

PONZONI, F.J.; SHIMABUKURO, Y.E.; KUPLICH, T.M. Sensoriamento remoto da vegetação. 2.ed. São Paulo: Oficina de Textos, 2012. 160p.

POURSANIDIS, D.; CHRYSOULAKIS, N.; MITRAKA, Z. Landsat 8 vs. Landsat 5: a comparison based on urban and peri-urban land cover mapping. International Journal of Applied Earth Observation and Geoinformation, v.35, p.259-269, 2015. DOI: 10.1016/j.jag.2014.09.010.

PRISHCHEPOV, A.V.; RADELOFF, V.C.; DUBININ, M.; ALCANTARA, C. The effect of Landsat ETM/ETM + image acquisition dates on the detection of agricultural land abandonment in eastern Europe. Remote Sensing of Environment, v.126, p.195-209, 2012. DOI: 10.1016/j. rse.2012.08.017.

SAMANIEGO, L.; SCHULZ, K. Supervised classification of agricultural land cover using a modified $k$-NN technique (MNN) and Landsat remote sensing imagery. Remote Sensing, v.1, p.875-895, 2009. DOI: 10.3390/rs1040875.

SISODIA, P.S.; TIWARI, V.; KUMAR, A. A comparative analysis of remote sensing image classification techniques. In: INTERNATIONAL CONFERENCE ON ADVANCES IN COMPUTING, COMMUNICATIONS AND INFORMATICS, 3., 2014, Delhi. Proceedings. New York: IEEE, 2014. p.1418-1421. DOI: 10.1109/ICACCI.2014.6968245.

TANSEY, K.; CHAMBERS, I.; ANSTEE, A.; DENNISS, A.; LAMB, A. Object-oriented classification of very high resolution airborne imagery for the extraction of hedgerows and field margin cover in agricultural areas. Applied Geography, v.29, p.145-157, 2009. DOI: 10.1016/j.apgeog.2008.08.004.

VALLE JÚNIOR, R.F. do; PISSARRA, T.C.T.; PASSOS, A. de O.; RAMOS, T.G.; ABDALA, V.L. Diagnóstico das áreas de preservação permanente na bacia hidrográfica do Rio Tijuco, Ituiutaba - MG, utilizando tecnologia SIG. Engenharia Agrícola, v.30, p.495-503, 2010. DOI: 10.1590/S0100-69162010000300013.

VIBRANS, A.C.; MCROBERTS, R.E.; MOSER, P.; NICOLETTI, A.L. Using satellite image-based maps and ground inventory data to estimate the area of the remaining Atlantic forest in Brazilian state of Santa Catarina. Remote Sensing of Environment, v.130, p.87-95, 2013. DOI: 10.1016/j.rse.2012.10.023.

WAHIDIN, N.; SIREGAR, V.P.; NABABAN, B.; JAYA, I.; WOUTHUYZEN, S. Object-based image analysis for coral reef benthic habitat mapping with several classification algorithms. Procedia Environmental Sciences, v.24, p.222-227, 2015. DOI: 10.1016/j.proenv.2015.03.029. 
WASKE, B.; VAN DER LINDEN, S.; BENEDIKTSSON, J.; RABE, A.; HOSTERT, P. Sensitivity of support vector machines to random feature selection in classification of hyperspectral data. IEEE Transactions on Geoscience and Remote Sensing, v.48, p.2880-2889, 2010. DOI: 10.1109/TGRS.2010.2041784.
ZHOU, W.; HUANG, G.; TROY, A.; CADENASSO, M.L. Object-based land cover classification of shaded areas in high spatial resolution imagery of urban areas: a comparison study. Remote Sensing of Environment, v.113, p.1769-1777, 2009. DOI: 10.1016/j.rse.2009.04.007.

Recebido em 18 de fevereiro de 2015 e aprovado em 21 de maio de 2015 